\title{
Primer registro de polillas perforadoras de frutos (Lepidoptera: Erebidae) de Colombia
}

\author{
First record of fruit piercing moths (Lepidoptera: Erebidae) in Colombia
}

\author{
JOSÉ MAURICIO MONTES RODRÍGUEZ1, HERNÁN DARÍO ROJAS² y NÉSTOR CESAY VACA BOHÓRQUEZ³
}

\begin{abstract}
Resumen: En plena época de cosecha, junio y julio de 2016, se presentó una inusual caída de frutos de mandarina y naranja que causó cuantiosas pérdidas en varios municipios productores del departamento de Santander, nororiente de Colombia. En algunos de los frutos se encontraron punciones, de las cuales se originaban pudriciones blandas; daño desconocido por los agricultores de la región. Muestreos nocturnos permitieron detectar dos especies de polillas: Eudocima apta y E. procus, realizando punciones en los frutos. La incidencia de los daños dejó de ser alta al desaparecer las polillas. Este es el primer reporte de polillas perforadoras de frutos en cultivos comerciales de naranja y mandarina en Colombia. Simultáneamente, a $60 \mathrm{~km}$ al oriente de los cultivos de cítricos, también se detectaron en el páramo de Berlín, municipio de Silos entre 3.000 a $3.100 \mathrm{msnm}$, lejos de cualquier huerto frutícola y de la planta hospedera registrada para E. apta, sugiriendo que se trataba de un paso migratorio.
\end{abstract}

Palabras clave: Neotropico, cítricos, Menispermaceae, Eudocima, migraciones.

\begin{abstract}
During the harvest season in June and July of 2016, there was an unusual drop of tangerine and orange fruits that caused heavy losses in several fruit producing municipalities in the department of Santander, in northeastern Colombia. In some of these fruits, punctures were found, from which soft rotting originated; this damage was unknown to farmers in the region. Nocturnal sampling allowed the detection of two species of fruit piercing moths, Eudocima apta and E. procus, puncturing the fruits. Moth damage changed from being high to disappearing within about two weeks. This is the first report of fruit piercing moths in orange and tangerine crops from Colombia. Simultaneously, 60 $\mathrm{km}$ east of the citrus crops, moths were also detected in the Berlin paramo in the municipality of Silos between 3,000 and 3,100 masl, far from any fruit orchard and from the host plants registered for E. apta, suggesting that this was a migratory move.
\end{abstract}

Key words: Neotropic, citrus, Menispermaceae, Eudocima, migrations.

\section{Introducción}

Las polillas del género Eudocima, subfamilia Calpinae (Erebidae), poseen una proboscis dura y esclerotizada adaptada para perforar y lacerar tejido, haciéndolas capaces de perforar frutos de plantas (Bänziger 1980; Zaspel et al. 2011). A diferencia de las otras polillas de importancia agrícola, las perforadoras de frutos realizan el daño en su estado adulto (Bhumannavar y Viraktamath 2012). En el estado larvario consumen el follaje de lianas silvestres de la familia Menispermaceae (Janzen y Hallwachs 2009). Al emerger el adulto puede volar a huertos frutícolas que se encuentran a grandes distancias debido a que posee una gran capacidad de vuelo (Bhumannavar y Viraktamath 2012).

Estos insectos son plagas de importancia agrícola en países asiáticos donde ocasionan cuantiosos daños. En cítricos, las polillas del género Eudocima pueden causar pérdidas hasta del $90 \%$ (Cochereau 1972; Bhumannavar y Viraktamath 2012). En algunas localidades son reportadas durante todo el año, con picos poblacionales que coinciden con la época de cosecha de cítricos (Ngampongsai et al. 2005; Leong y Kueh 2011), y las prácticas de manejo que se conocen son poco efectivas y costosas (Bhumannavar y Viraktamath 2012). Eudocima phalonia (Linnaeus, 1763) la especie más importante del grupo, tiene la capacidad de perforar frutos de más de 100 especies de plantas en 34 familias (Davis et al. 2005; Gilligan y Passoa 2016).

En contraste, en el continente americano, solo existe un registro de daño por polillas del género Eudocima en cultivos de papaya (Hernández-Ruiz et al 2017). La información básica de biología y ecología es desconocida para la mayoría de las especies y pocos muestreos se han realizado para conocer su diversidad y distribución (Zenker et al. 2012). Por ejemplo, de las ocho especies del género Eudocima registradas en el continente americano sólo se conoce la planta hospedera de tres de ellas (Janzen y Hallwachs 2009; Robinson et al. 2010).

El daño realizado por las polillas perforadoras de frutos se caracteriza por una punción de la cual se origina una pudrición generalmente acompañada de la caída de los frutos, los cuales son invadidos por dípteros y coleópteros saprófagos (Norris 1935; Todd 1959). Daños de este tipo se encontraron en junio y julio de 2016 en la región citrícola del departamento de Santander, en el nororiente de Colombia. Las pérdidas no se cuantificaron, sin embargo, una parte considerable de la cosecha fue afectada y los agricultores preocupados con esta situación, recurrieron a instituciones públicas del sector agrícola para pedir ayuda. El diagnóstico de este problema fitosanitario fue el principal objetivo que motivó este trabajo.

\footnotetext{
${ }^{1}$ M. Sc. Ciencias - Entomología. Corporación Colombiana de Investigación Agropecuaria - AGROSAVIA. Centro de Investigación La Suiza - Km 32 vía al mar, vereda Galápagos, Rionegro, Santander, Colombia, jmontesr@corpoica.org.co. Código Orcid: https://orcid.org/0000-0002-9281-2991. ${ }^{2}$ Ingeniero Agrónomo. Instituto Colombiano Agropecuario ICA. Subgerencia de protección vegetal. Avenida quebrada seca 31-39, A. A. 1017, Bucaramanga, Santander, Colombia, hdrojas@unal.edu.co. ${ }^{3}$ Asociación de productores agrícolas sostenibles de Rionegro FRUTROPS, Km 23 vía al mar, Bodega 2, Rionegro, Santander, Colombia, frutrops2013@gmail.com. Autor para correspondencia: José Mauricio Montes Rodríguez. M. Sc. Ciencias - Entomología. Corporación Colombiana de Investigación Agropecuaria - AGROSAVIA. Centro de Investigación La Suiza - Km 32 vía al mar, vereda Galápagos, Rionegro, Santander, Colombia,jmontesr@corpoica.org.co.
} 


\section{Materiales y métodos}

Los muestreos se realizaron en la finca La Esperanza, vereda La Paz, en el municipio de Rionegro, departamento de Santander, Colombia, 7¹9'28,8'N 73¹0'30,2”O, a 1.105 msnm, del 1 al 5 de julio. Por esos días, el daño a los frutos en cultivos de naranja y mandarina en la región era alto. Se visitaron cultivos de cítricos en las primeras horas de la noche (18:30 a 20:30 h), período que es el de mayor actividad de las polillas perforadoras de frutos (Gilligan y Passoa 2016). Se inspeccionaron frutos de naranja y mandarina en busca de polillas perforadoras de frutos, las cuales se capturaron con red entomológica, a los especímenes se les inyectó alcohol en el abdomen y se refrigeraron $\left(2\right.$ a $\left.6{ }^{\circ} \mathrm{C}\right)$ hasta su montaje en seco.

Como complemento a los muestreos nocturnos en los cultivos de cítricos, se hicieron observaciones de las polillas perforadoras de frutos en el páramo de Berlín, aproximadamente 60 kilómetros al oriente de los cultivos mencionados. En las primeras horas de la mañana se inspeccionó cerca de las fuentes de luz de las casas de esta zona rural en el municipio de Silos, Norte de Santander. Se realizaron visitas quincenales entre abril y septiembre de 2016. Debido a su pertinencia se decidió incluir está información en el análisis.

Las muestras se identificaron con la información taxonómica disponible para el género Eudocima utilizando principalmente el patrón de coloración de las alas (Zilli y Hogenes 2002). En unos especímenes se extrajo la genitalia, para lo cual, se disectó el abdomen y se colocó en hidróxido de potasio a temperatura ambiente durante 24 horas, posteriormente, se limpió con agua destilada y fue almacenada en glicerina, después de estar 30 minutos en etanol al $70 \%$. El material colectado se depositó en la colección taxonómica nacional de insectos "Luis María Murillo” (CTNI).

\section{Resultados y discusión}

Se recolectaron dos especies de polillas (Lepidoptera: Erebidae) afectando los frutos de cítricos identificadas como Eudocima apta (Walker, [1858] 1857) y Eudocima procus (Cramer, 1777) (Figs. 1A y B, respectivamente). Es la primera vez que ambas especies se registran perforando frutos de cítricos en Colombia y en Suramérica. En México E. apta fue reportada afectando frutos de papaya junto a $E$. serpentifera (Hernández-Ruiz et al. 2017).

La distribución geográfica es amplia para las dos especies. E. apta aunque neotropical (Zilli y Hogenes 2002), también es reportada en Estados Unidos y Canadá donde es con- siderada una especie errante o accidental (Gilligan y Passoa 2016) o migrante tropical ocasional (Brou 2006). En islas del Atlántico, los registros son basados en uno o pocos adultos colectados o encontrados en colecciones por lo que igualmente podrían tratarse de registros de individuos errantes o accidentales (Zagatti et al. 2006; Brou y Núñez 2013; Brou et al. 2013). En el caso de E. procus, los registros se restringen a Suramérica en Colombia, Brasil y Surinam (Specht y Corseuil 2002; Zaspel y Branham 2008).

Material examinado. Eudocima procus. $1 q 1 \AA$. COLOMBIA. Norte de Santander. Silos. Páramo de Berlín. Sector La Laguna. $7^{\circ} 14^{\prime} 16,1^{\prime \prime N} 72^{\circ} 48^{\prime} 37^{\prime \prime} O .3171$ m. Captura manual. 2-jul-2016. José Montes. [CTNI]. 1ð. Santander. Rionegro. vereda La Paz. Finca La Esperanza. $7^{\circ} 19^{\prime} 28,8^{\prime \prime} \mathrm{N}$ 73¹0’30,2”O. 1105 msnm. 5-jul- 2016. Captura manual. Néstor Vaca [CTNI].

Eudocima apta. 1910 . COLOMBIA. Norte de Santander. Silos. Páramo de Berlín. Sector La Laguna. 7²14'16,1”N 7248'37'O. 3171 msnm. Captura manual. 2-jul-2016. José Montes. [CTNI]. $1 q$ Santander. Rionegro. Vereda La Paz. Finca La Esperanza. 7¹9'28,8'N 73¹0’30,2”O. 1105 msnm. Captura manual. 5-jul-2016. Hernán Rojas. [CTNI].

Posterior a la recolecta, se verificó que las polillas hicieran exactamente el mismo daño que inicialmente habían reportado los agricultores, caracterizado por una punción circular de la cual emergían gotas de jugo. Las polillas E. apta y E. procus siguen una secuencia de perforación del fruto similar al de otras especies de la subfamilia Calpinae (Fennah 1942; Bazinger 1980). Inicialmente la mariposa se ubica en el fruto y lo perfora con la proboscis, cerca de las patas anteriores. Una vez logra perforar, empuja e introduce buena parte de la proboscis, mientras mueve oscilatoriamente las antenas. En este proceso demora unos pocos minutos. Hay frutos en los cuales se detectaron hasta cuatro perforaciones.

En el área citrícola de Santander, se encontró evidencia de este daño en frutos de naranja y mandarina, desde principios de junio hasta el 15 de julio de 2016, disminuyendo drásticamente a partir de entonces. Aunque no se puede descartar que este daño hubiera ocurrido en el pasado, no fue notable en años anteriores. La variación anual de las poblaciones de las polillas del género Eudocima ha sido relacionada principalmente con los períodos de lluvia y sequía (Hargreaves 1936; Leong y Kueh 2011). Sin embargo, se desconocen los factores que favorecen incrementos poblacionales y daños en solo unos años en particular, como ocurrió en los cítricos de Santander en Colombia y en cultivos de papaya en México (Hernández-Ruiz et al. 2017).

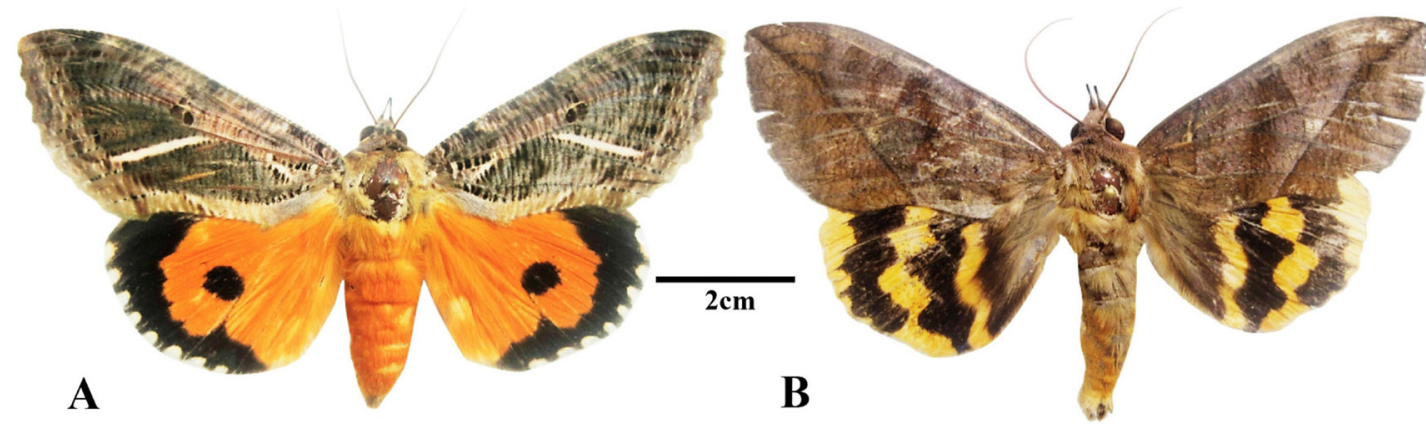

Figura 1. Polillas perforadoras de frutos en cítricos en el municipio de Rionegro, Santander. A. Eudocima apta. B. Eudocima procus. 
Un evento similar al reportado en el departamento de Santander fue documentado por Cochereau $(1972 ; 1977)$ en la isla de Nueva Caledonia al oriente de Australia, al estudiar las polillas perforadoras de frutos de la especie $E$. phalonia, durante varios años. El autor registró incrementos poblacionales esporádicos que duraban unas pocas semanas y que afectaban los cultivos de cítricos y otros frutales ocasionando pérdidas de la producción de más del $90 \%$, cuando en un período normal el daño por la punción de las polillas era del $4 \%$.

Cuando se presentan estos incrementos poblacionales es difícil su manejo y las medidas que se han ensayado son poco eficientes o muy costosas para ser seguidas (Cochereau 1972; Bhumannavar y Viraktamath 2012). Sin embargo, se espera que prácticas culturales como la recolección de frutos muy maduros del suelo y la cosecha oportuna disminuyan las pérdidas, debido a que las polillas muestran preferencia por frutos maduros, además se reduce el tiempo de exposición de los frutos (Mote et al. 1991; Hernández-Ruiz et al. 2017). El momento de llegada de las polillas coincidió con la cosecha y un precio bajo de la mandarina, esto propició que muchos agricultores no cosecharán oportunamente, generando alta disponibilidad de frutos para las mariposas, agravando el problema.

Por otra parte, en el páramo de Berlín se encontraron las dos especies de polillas, desde el 18 de junio hasta el 30 de julio, aunque la mayor cantidad $(90 \%)$ de especímenes se detectó en los días 2 y 16 de julio. En este ambiente a 3.100 msnm donde predomina vegetación de páramo, pasturas y hortalizas, no se espera que se establezcan estas polillas, debido a la falta de huertos frutícolas de los cuales se alimentan los adultos y la ausencia de la planta hospedera conocida para $E$. apta, que es la liana silvestre Disciphania heterophylla Barneby (Menispermaceae), la cual está registrada en Perú, Bolivia, Costa Rica y Brasil en alturas que no superan los $650 \mathrm{msnm}$ (León et al. 2006; Janzen y Hallwachs 2009). Todo lo anterior sugiere que se trató de un paso migratorio.

En el oriente de México, Powell y Brown (1990) encontraron a Eudocima apta junto con otras especies de las familias Noctuidae y Sphingidae típicas de tierras bajas en zona montañosa a $2.350,2.700$ y $3.900 \mathrm{msnm}$, fuera del rango de distribución de sus plantas hospederas, ellos se unieron a la hipótesis ya planteada por Janzen (1987) en Costa Rica, quien sugiere que estas migraciones y concentraciones de polillas en alta montaña pueden ser influenciadas por factores como la presión de los enemigos naturales y la disponibilidad de alimento, que motivan la migración de algunas especies de mariposas con capacidad de volar grandes distancias como E. apta.

El daño reportado en la cosecha de cítricos de Santander en 2016 por E. apta y E. procus puede considerarse un evento inusual, con muchos interrogantes de su biología y ecología por resolver. De esta manera, más muestreos son necesarios en especial en áreas silvestres en busca de los estados inmaduros y de sus plantas hospederas en Colombia, acompañados de muestreos nocturnos en huertos frutícolas. Poco a poco esta información permitirá confirmar la capacidad migratoria de sus poblaciones y predecir en que momento volverán a la región citrícola de Santander para estar preparados ante estos visitantes ocasionales.

\section{Agradecimientos}

Los autores quieren expresar sus agradecimientos a Román Galeano y demás agricultores que colaboraron de una u otra manera en el montaje de los ensayos; a Corina Buendía Grigoriu, Andrés Iván Prato y los demás revisores por sus valiosos aportes y sugerencias. Este trabajo fue financiado con recursos públicos de la Corporación Colombiana de Investigación Agropecuaria (Corpoica), y del Instituto Colombiano Agropecuario (ICA).

\section{Literatura citada}

BÄNZIGER, H. 1980. Skin-piercing blood-sucking moths III: Feeding act and piercing mechanism of Calyptra eustrigata (Hmps.) (Lepidoptera: Noctuidae). Bulletin de la Société Entomologique Suisse 53: 127-142.

BHUMANNAVAR, B. S.; VIRAKTAMATH, C. A. 2012. Biology, ecology and management of fruit piercing moths (Lepidoptera: Noctuidae). Pest Management in Horticultural Ecosystems 18 (1): $1-18$.

BROU JR., V. A. 2006. A new US record for the tropical fruit-piercing moth Eudocima serpentifera (Walker, 1858). Southern Lepidopterists News 28: 105-108.

BROU JR., V. A.; NÚÑEZ A., R. 2013. Eudocima toddi (Zayas, 1965) (Lepidoptera: Erebidae) a rare and endemic species of Cuba. Southern Lepidopterists News 35: 92-93.

BROU JR., V. A.; GIESE, A. R.; MILLER, D. H. 2013. A new U.S. state record for a tropical fruit-piercing moth in the family Erebidae Leach. Southern Lepidopterists News 35: 27-28.

COCHEREAU, P. 1972. Population management of the fruit sucking moth Othreis fullonia (Clerck) in New Caledonia. 14th International Congress of Entomology (Canberra). Disponible en: http://horizon.documentation.ird.fr/exl-doc/pleins textes/ pleins_textes_5/b_fdi_04-05/06156.pdf. [Fecha revisión: 6 agosto 2017].

COCHEREAU, P. 1977. Biologie et écologie des populations en Nouvelle-Calédonie d'un papillon piqueur de fruits: Othries fullonia Clerck (Lepidoptera, Noctuidae, Catocalinae). Travaux et Documents ORSTOM No. 71. 322 p.

DAVIS, E. E.; FRENCH, S.; VENETTE, R. C. 2005. Mini risk assessment - fruit piercing moth: Eudocima fullonia Green [Lepidoptera: Noctuidae]. USDA-CAPS, 43 p.

FENNAH, R. G. 1942. The citrus pests investigation in the windward and leeward islands, British West Indies, 1937-1942. 66 p.

GILLIGAN, T. M.; PASSOA, S. C. 2016. Screening aid fruit piercing moth Eudocima phalonia (Linnaeus) Lepidoptera: Erebidae. United States Department of Agriculture. Disponible en: http://caps.ceris.purdue.edu/taxonomic_services. [Fecha revisión: 7 agosto 2016].

HARGREAVES, E. 1936. Fruit-piercing Lepidoptera in Sierra Leone. Bulletin of Entomological Research 27: 589-605.

HERNÁNDEZ-RUIZ, A.; ILLESCAS-RIQUELME, C. P.; BAUTISTA-MARTÍNEZ, N. VARGAS-ABASOLO, R.; VALDEZCARRASCO, J. M.; FIGUEROA-CASTRO, P. 2017. Identification of fruit-piercing moths (Lepidoptera: Erebidae) and damage caused to papaya fruit in Mexico. Entomological News 126 (5): 415-420.

JANZEN, D. H. 1987. When, and when not to leave. Oikos 49 (3): 241-243.

JANZEN, D. H.; HALLWACHS, W. 2009. Dynamic database for an inventory of the macrocaterpillar fauna, and its food plants and parasitoids, of area de conservacion Guanacaste (ACG), northwestern Costa Rica. Disponible en: http://janzen.sas.upenn. edu [Fecha revisión: 1 noviembre 2016].

LEÓN, B.; PITMAN, N.; ROQUE, J. 2006. Introducción a las plantas endémicas del Perú. Revista Peruana de Biología. Número especial 13 (2): 9-22.

LEONG, S. C. T.; KUEH, R. J. H. 2011. Seasonal abundance and suppression of fruit-piercing moth Eudocima phalonia (L.) in a citrus orchard in Sarawak. The Scientific World Journal 11: $2330-2338$. 
MOTE, U. N.; TAMBE, A. B.; PATIL, C. S. 1991. Observation on incidence and extent of damage of fruit sucking moths on pomegranate fruits. Journal of Maharashtra Agricultural Universities 16 (3): 438-439.

NGAMPONGSAI, A.; BARRETT, B.; PERMKAM, S.; SUTHAPRADIT, N.; NILLA-OR, R. 2005. A preliminary study on some ecological aspects of the fruit piercing moths in Songkhla Province of Southern Thailand Songklanakarin. Journal of Science and Technology 27 (6): 1135-1145.

NORRIS, M. J. 1935. The feeding habits of the adult Lepidoptera Heteroneura. Transactions of the Royal Entomological Society of London 85: 61-90.

POWELL, J. A.; BROWN, J. W. 1990. Concentrations of lowland sphingid and noctuid moths at high mountain passes in eastern Mexico. Biotropica 22 (3): 316-319.

ROBINSON, G. S.; ACKERY, P. R.; KITCHING, I. J.; BECCALONI, G. W.; HERNÁNDEZ, L. M. 2010. HOSTS - A database of the world's lepidopteran hostplants. Natural History Museum, London. Disponible en: http://www.nhm.ac.uk/hosts. [Fecha revisión: 1 noviembre 2016].

SPECHT, A.; CORSEUIL, E. 2002. Diversidade dos noctuídeos (Lepidoptera, Noctuidae) em Salvador do Sul, Rio Grande do Sul, Brasil. Revista Brasilera de Zoologia 19 (1): 281-298.

TODD, E. L. 1959. The fruit piercing moths of the genus Gonodonta Hübner (Lepidoptera: Noctuidae). Technical Bulletin $\mathrm{N}^{\circ}$ 1201, Agricultural Research Service. United States of Agriculture. Washington, D. C. 69 p.

ZAGATTI, P.; LALANNE-CASSOU, B.; DUCHAT D'AUBIGNY, J. 2006. Catalogue of the Lepidoptera of the French Antilles.
Institut National de la Recherche Agronomique. Disponible en: http://www7.inra.fr/papillon/indexeng.htm. [Fecha revisión: 1 noviembre 2016].

ZASPEL, J. M.; BRANHAM, M. A. 2008. World checklist of tribe Calpini (Lepidoptera: Noctuidae: Calpinae). Insecta Mundi 47: 1-16.

ZASPEL, J. M.; WELLER, S. J.; BRANHAM, M. A. 2011. A comparative survey of proboscis morphology and associated structures in fruit-piercing, tear-feeding, and blood-feeding moths in Calpinae (Lepidoptera: Erebidae). Zoomorphology 130: 203225

ZENKER, M. M.; BOTTON, M.; SPECHT, A.; MOSER, A. 2012. Mariposas em parreirais na região da Serra Gaúcha e informações sobre espécies com potencial para perfurar frutos. Circular técnica 89. Ministério da Agricultura, Pecuária e Abastecimento. $8 \mathrm{p}$

ZILLI, A.; HOGENES, W. 2002. An annotated list of the fruit-piercing moth genus Eudocima Blllberg, 1820 (sensu Poole) with descriptions of four new species (Lepidoptera: Noctuidae, Catocalinae). Quadrifina 5: 153-207.

Recibido: 25-feb-2017 - Aceptado: 09-nov-2017

Citación sugerida:

MONTES RODRÍGUEZ, J. M.; ROJAS, H. D.; VACA BOHÓRQUEZ, N. C. 2018. Primer registro de polillas perforadoras de frutos (Lepidoptera: Erebidae) de Colombia. Revista Colombiana de Entomología 44 (1): 116-119. Enero - Junio 2018. 